\title{
Commentary
}

\section{Reactive Oxygen Species in Disease: Rebuttal of a Conventional Concept}

\author{
Luis Vitetta ${ }^{1,2}$, Samantha Coulson ${ }^{1,2}$, Anthony W Linnane ${ }^{1,2}$ \\ ${ }^{1}$ The University of Sydney, Sydney Medical School - Medical Sciences, Discipline of Pharmacology, \\ Australia; 2 Medlab Clinical Ltd., Sydney, Australia.
}

\begin{abstract}
The production of intracellular reactive oxygen species and reactive nitrogen species has long been proposed as leading to the random deleterious modification of macromolecules (i.e., nucleic acids, proteins) with an associated progressive development of the age associated systemic diseases (e.g., diabetes, Parkinson's disease) as well as contributing to the ageing process. Superoxide anion (hydrogen peroxide) and nitric oxide (peroxynitrite) comprise regulated intracellular second messenger pro-oxidant systems, with specific sub-cellular locales of production and are essential for the normal function of the metabolome and cellular electro-physiology. We have posited that the formation of superoxide anion and its metabolic product hydrogen peroxide, and nitric oxide, do not conditionally lead to random damage of macromolecular species such as nucleic acids or proteins. Under normal physiological conditions their production is intrinsically regulated that is very much consistent with their second messenger purpose of function. We further propose that the concept of an orally administered small molecule antioxidant as a therapy to abrogate free radical activity (to control oxidative stress) is a chimera. As such we consider that free radicals are not a major overwhelming player in the development of the chronic diseases or the ageing process.
\end{abstract}

Received: 24 August 2015; Accepted after revision: 03 September 2015; Published: 07 September 2015.

Author for correspondence: Luis Vitetta, The University of Sydney, Sydney Medical School - Medical Sciences, Discipline of Pharmacology, Australia, E-mail: luis.vitetta@sydney.edu.au

How to cite: Vitetta L, Coulson S, Linnane AW. Reactive Oxygen Species in Disease: Rebuttal of a Conventional Concept. Journal of Controversies in Biomedical Research 2015; 1(1):23-27.

Doi: http://dx.doi.org/10.15586/jcbmr.2015.7

Licence: This open access article is licenced under Creative Commons Attribution 4.0 International (CC BY 4.0). http://creativecommons.org/licenses/by/4.0/

\section{Introduction}

Numerous compounds that have been isolated from foods, nutrients and herbal medicines, commonly defined as antioxidants, have been advanced as molecules that can effectively counterbalance the over-production of free radicals produced by intracellular oxidation reactions in the expectation of ameliorating symptoms of the chronic diseases. It has been reported that the body can function effectively with low levels of free radicals but if there is an overload of reactive oxygen species (ROS) and or reactive nitrogen species (RNS) that then there is an increased risk for diabetes, chronic kidney disease, heart disease, cancer, and other chronic diseases. We have challenged the antioxidant-free radical theory of chronic diseases and ageing and advanced the idea that there is a critical requirement for free radicals that is consistent with their intracellular second messenger functions 
(1-3), a fact that is rarely acknowledged in such studies.

Antioxidant supplements are among the most popular over-the-counter health products in the world with a net worth of billions of dollars in global sales without the requirement of a health practitioner's prescription $(4,5)$. The motivation for this influx of high market value is largely based on in vitro research models conducted to demonstrate that free radicals could be counterbalanced suggesting that antioxidant compounds in foods and supplements could benefit health by abrogating the over production of free radicals.

There has been a plethora of investigations on antioxidants and the rescuing of the asserted oxidative damage to macromolecules that ensues. However the conclusions emanating from the in vitro and in vivo experimental models have no relevance to normal physiological function and therefore the significance to the risk of developing a chronic disease or affecting the ageing process is a null effect (1). Hydrogen peroxide is the immediately obvious substance employed in such investigations with thousands of articles in the medical and scientific literature reporting it as causal for macromolecular damage and severe cellular toxicity. The demonstration of cellular toxicity is based on employing hydrogen peroxide levels that far exceed those observed under normal physiological conditions, otherwise it is cited that at lower levels the phenomenon is not detectable. The hypothesis that is advanced and justified is that the high levels of hydrogen peroxide are requisite in order to demonstrate the toxic effect that hydrogen peroxide has on cells and its complement of macromolecules. Additional experiments investigating the levels of cellular catalase and glutathione peroxidase, which function to regulate the level of hydrogen peroxide, further postulate that these enzyme systems prevent the cellular damage that hydrogen peroxide may cause. In contrast to this, hydrogen peroxide is not a toxic compound at physiological levels (1).

This commentary discusses in brief the role of these second messengers in the regulation of the metabolome in terms of radical formation as an essential contributor to the physiologically normal regulation of sub-cellular bioenergy systems; proteolysis regulation; transcription activation; enzyme activation; mitochondrial DNA changes; redox regulation of metabolism and cell differentiation (1).

\section{Historical view and contradictions}

Historically, Harman (6) in 1956 hypothesized that free radicals (also termed oxygen radical formation) was a major injurious contributor to the ageing process and degenerative diseases due to their attack on cell constituents (macromolecules such as DNA, proteins, cell membranes) and connective tissues. An extensive set of experiments followed that received strong support for the hypothesis that free radicals were causal for oxidative damage. Boveris and Chance (7) in 1973 showed that large amounts of superoxide anions were generated by the mitochondrial electron transport chain during the phase of oxidative phosphorylation of the Kreb's cycle, whereby complex I and II reduced coenzyme Q10 and its oxidation by complex III. Furthermore, Chance and colleagues (8) then reported in 1979 and estimated that 1$3 \%$ of inspired oxygen was converted to ROS, which would indeed be toxic to cells. Chance's experiments however, were contradicted when Staniek and Nohl (9) in 2000 and St-Pierre and colleagues (10) in 2002 demonstrated that intact normally respiring mitochondria were not observed to produce high concentrations of ROS and that the earlier extrapolations of superoxide anion and hydrogen peroxide were over estimations by several orders of magnitude. Therefore, the high amounts of ROS were not produced in physiologically normally respiring human mitochondria and that the human cellular metabolome tightly regulates the production of ROS/RNS. This then indicating that oxidative damage did not occur unless the system was induced to do so in a non-physiological experimental setting.

\section{Is oxidative damage involved in depressive disorder?}

In a recent review by Tobe (11), investigations on mitochondrial dysfunction 
and oxidative damage in major depressive disorder were considered. The human studies described in that review were based on brain imaging captured via magnetic resonance imaging and post mortem histologic studies. It was suggested that the decreased size of the brain, decreased glial cell density and neuronal size linked with major depressive disorder, biopolar or schizophrenia were due to oxidative stress. There was no scientific or mechanistic explanation that confirmed this suggestion nor was it associated with a systemic overproduction of free radicals. The basis of the discussion was reverted to animal studies, which had established a nonphysiological environment that was causal for increased ROS production, inhibition of mitochondrial respiration and oxidative DNA damage (12-14). Such investigations reinforce and reiterate that oxidative damage does not occur under normal physiological conditions. The cellular damage alluded to in those investigations occurs in a set of experiments with induced abnormal productions of ROS/RNS.

\section{Is antioxidant therapy beneficial?}

Additional recent experiments by others (15) have questioned the role of antioxidant supplementations, suggesting that introduction of antioxidants may cause antioxidant-induced stress whereby these compounds overwhelm the body's free radical production disrupting cellular redox balance. Many investigators report benefits of antioxidant administration however there are only a few that question the possible harmful effects (16). Free radicals have been designated and repeatedly reported as largely harmful chemical entities and as such have a negative impact on cellular metabolism and mitochondria and therefore are causal for macromolecular oxidative damage (16). However, it has been reported that ROS and RNS participate in specific functions and play an important role in signal transduction in many physiological events $(1-3,9,10)$. ROS and RNS play a significant role in signal transduction of cytokine receptors, tyrosine receptors, serine/threonine kinases, G proteincoupled receptors, ion-channel linked receptors in response to angiotension II, cytokines, glutamate, epidermal growth factor, vascular endothelial growth factor, tumour necrosis factor alpha and platelet derived growth factor $(15,16)$. Furthermore, hydrogen peroxide is a known mitogen (1).

Villanueva and Kross (15) form the argument that excess consumption of administered antioxidants can overwhelm the cellular function of ROS / RNS and therefore decrease their biological function within cells. This deleterious action then goes on to interfere with the normal cellular processes and requirements by disrupting biochemical and physiological activity required for normal cellular function. They list nine trials indicating no effect from antioxidant supplementation and six trials that found harmful effects from antioxidant supplementation versus twelve that reported a benefit (15). This body of evidence raises a further query and concern as to antioxidant supplementation benefits versus safety.

The question may not only be if antioxidant supplementation is beneficial or detrimental but is there an antioxidant effect? What has been reported is that antioxidants that are referred to in the literature can also be designated as pro-oxidants, inducing the formation of hydrogen peroxide, a necessary biochemical requisite for optimal cellular function. It would hence seem that antioxidants promote healthy cellular metabolism by providing a regulated oxidoreductase activity. Coenzyme Q10 is such an example. Coenzyme Q10 is a key component of the oxidative phosphorylation section of energy production and electron transport chain as well as other organelle oxido-reductase activity (3). Coenzyme Q10 has been labeled as a strict antioxidant however it also has a pro-oxidant function through the formation of superoxide anion and hydrogen peroxide that is a major factor in its beneficial mode of activity (3).

\section{Reprise}

The human metabolome is an expression of a finely tuned dynamic equilibrium, which is comprised of thousands of anabolic and catabolic reactions and all cellular systems are finely regulated. However, there is no perfect machine and malfunctions can occur (1). If there is a small inappropriate leakage of free radicals, the mitochondria or cell can be damaged and will go into 
apoptosis (death) and cannot continue to function in a compromised state as suggested by some authors $(17,18)$. Currently, there are no blinded human clinical trials establishing excessive systemic over production of reactive oxygen species as the primary cause of aging or associated diseases (1).

The canon belief that the production of ROS and RNS leads to random deleterious modification of macromolecular species, mitochondria and cellular metabolism and that oxidative damage is a major contributor to ageing and related systemic diseases is untenable. Furthermore, the administration of antioxidants such as vitamin A, C, E, compounds found in herbs or coenzyme Q10 that can ameliorate oxidative stress is flawed. ROS and RNS are products of normal cellular metabolism and are necessary for normal physiological functioning of the organism.

Furthermore, consider as an extreme malfunction of the superoxide anion/NO systems that are often cited in support of the free radical damage theory. The first, is septic shock which affects a small number of individuals arises from phagocytic cells over producing the radicals consequent on a microbial infection that leads to an unrelenting and life taking arterial hypotension unless the causative infection is resolved. Secondly, the alternating view is exemplified by chronic granulomatous disease, a rare immuno-deficiency disease arising from a phox 67 mutation that is characterized by the inability of neutrophils to adequately respond to a microbial infection. The cells of the immune system under produce superoxide anion/nitric oxide and most patients die from overwhelming infections at an early age as a result of the deficit.

\section{Conclusion}

Antioxidants can also act as pro-oxidants (e.g. ascorbic acid) therefore readdressing the action of these molecules to oxidoreductase molecules may serve researchers with a more appropriate mode of action for further investigations of efficacy. The antioxidant compounds marketed still play a vital role and should be included in a prescription of health. However, additional clinically relevant research is required though, that considers that the evolutionary progression of humans has become dependent upon the production of ROS and RNS formation. Reassessment of the antioxidant theory and a new paradigm of thinking are certainly required.

\section{Conflict of Interest}

Luis Vitetta has received National Institute of Complementary Medicine and National Health and Medical Research Council of Australia competitive funding and Industry support for research into nutraceuticals and herbal medicines. The authors declare no other potential conflicts of interest with respect to research, authorship and/or publication of this article.

\section{References}

1. Linnane AW, Kios M, Vitetta L. Healthy aging: Regulation of the metabolome by cellular redox modulation and prooxidant signaling systems: The essential roles of superoxide anion and hydrogen peroxide. Biogerontology 2007;8(5):445-467.

http: / /dx.doi.org/10.1007/s10522-0079096-4

PMid: 17415678

2. Linnane AW, M. Kios, Vitetta L. The essential requirement for superoxide radical and nitric oxide formation for normal physiological function and healthy aging. Mitochondrion 2007;7(1-2):1-5. http:/ /dx.doi.org/10.1016/j.mito.2006.11. 009

PMid:17317335

3. Linnane AW, Kios M, Vitetta L. Coenzyme Q(10)--its role as a prooxidant in the formation of superoxide anion/hydrogen peroxide and the regulation of the metabolom. Mitochondrion 2007; 7:S51-61. http: / /dx.doi.org/ 10.1016/j.mito.2007.03. 005

PMid:17482887

4. Choices. 2011 Supplements who needs them? NHS. June: 1-33.

5. Saeidnia S, Abdollahi M. Toxicological and pharmacological concerns on oxidative stress and related diseases. Toxicol Appl 
Pharmacol 2013;273(3):442-55.

http://dx.doi.org/ 10.1016/j.taap.2013.09. 031

6. Harman D. Aging: a theory based on free radical and radiation chemistry. J Gerontol. 1956;11(3):298-300.

http://dx.doi.org/10.1093/geronj/11.3.29

8

PMid:13332224

7. Boveris A, Chance B. The mitochondrial generation of hydrogen peroxide. General properties and effect of hyperbaric oxygen. Biochem J 1973;134(3):707-16.

http:/ /dx.doi.org/ 10.1042/bj 1340707

PMid:4749271 PMCid:PMC1177867

8. Chance B, Sies H, Boveris A. Hydroperoxide metabolism in mammalian organs. Physiol Rev 1979;59(3):527-605.

PMid:37532

9. Staniek K, Nohl H. Are mitochondria a permanent source of reactive oxygen species? Biochim Biophys Acta 2000;460(23):268-75.

http:/ /dx.doi.org/10.1016/S0005-

2728(00)00152-3

10. St-Pierre J, Buckingham JA, Roebuck SJ, Brand MD. Topology of superoxide production from different sites in the mitochondrial electron transport chain. J Biol Chem 2002; 277(47):44784-90. http://dx.doi.org/ 10.1074/jbc.M20721720 0

PMid:12237311

11. Tobe EH. Mitochondrial dysfunction, oxidative stress, and major depressive disorder. Neuropsychiatr Dis Treat 2013; 9:567-73.

http:/ / dx.doi.org/ 10.2147/NDT.S44282

PMid:23650447 PMCid:PMC3640606

12. Lee HM, Reed J, Greeley GH Jr, Englander EW. Impaired mitochondrial respiration and protein nitration in the rat hippocampus after acute inhalation of combustion smoke. Toxicol Appl Pharmacol 235(2):208. http://dx.doi.org/10.1016/j.taap.2008.12. 010

PMid:19133281 PMCid:PMC2967486

13. Lee HM, Greeley GH, Herndon DN, Sinha M, Luxon BA, Englander EW. A rat model of smoke inhalation injury: influence of combustion smoke on gene expression in the brain. Toxicol Appl Pharmacol 2005;208(3):255-65.

http://dx.doi.org/10.1016/j.taap.2005.03. 017

PMid:15893353

14. Lee HM, Greeley GH Jr., Englander EW. Transgenic overexpression of neuroglobin attenuates formation of smoke-inhalationinduced oxidative DNA damage, in vivo, in the mouse brain. Free Radic Biol Med 2011; 51(12):2281-7.

http://dx.doi.org/10.1016/j.freeradbiomed .2011 .09 .026

PMid:22001746 PMCid:PMC3241998

15. Villanueva C, Kross R. Antioxidantinduced stress. Int J Mol Sci 2012; 13(2): 2091-109.

http:/ / dx.doi.org/ 10.3390/ijms13022091

PMid:22408440 PMCid:PMC3292009

16. Valko M, Leibfritz D, Moncol J, Cronin MT, Mazur M, Telser J. Free radicals and antioxidants in normal physiological functions and human disease. Int $\mathrm{J}$ Biochem Cell Biol 2007;39(1):44-84.

http://dx.doi.org/10.1016/j.biocel.2006.0 7.001

PMid:16978905

17. Parikh SM. Therapeutic targeting of the mitochondrial dysfunction in septic acute kidney injury. Curr Opin Crit Care 2013;19(6):554-9.

http:/ / dx.doi.org/ 10.1097/MCC.00000000 00000038

18. Muyderman H, Chen.T. Mitochondrial dysfunction in ALS - a valid pharmacological target? Br J Pharmacol 2014;171(8):2191205.

http://dx.doi.org/10.1111/bph.12476 